\title{
A genome-wide association study of the association between single nucleotide polymorphisms and brachial-ankle pulse wave velocity in healthy Koreans
}

\author{
EnShi Xu', Jinho Shin, ${ }^{1, *}$ Ji Eun Lim² ${ }^{1}$ Mi Kyung Kim ${ }^{3,4}$, Bo Youl Choi, ${ }^{3,4}$, Min-Ho Shin ${ }^{5}$, Dong Hoon Shin ${ }^{6}$, Young-Hoon Lee, \\ Byung-Yeol Chun ${ }^{8,9}$, Kyung-Won Hong ${ }^{10}$, and Joo-Yeon Hwang ${ }^{10}$ \\ 'Department of Internal Medicine, Hanyang University College of Medicine, Seoul, Korea \\ ${ }^{2}$ Department of Biochemistry and Molecular Biology, Kyung Hee University School of Medicine, Seoul, Korea \\ ${ }^{3}$ Department of Preventive Medicine, Hanyang University College of Medicine, Seoul, Korea \\ ${ }^{4}$ Institute for Health and Society, Hanyang University, Seoul, Korea \\ ${ }^{5}$ Department of Preventive Medicine, Chonnam National University Medical School, Gwangju, Korea \\ ${ }^{6}$ Department of Preventive Medicine, Keimyung University School of Medicine, Daegu, Korea \\ ${ }^{7}$ Department of Preventive Medicine \& Institute of Wonkwang Medical Science, Wonkwang University School of Medicine, Iksan, Korea \\ ${ }^{8}$ Department of Preventive Medicine, Kyungpook National University School of Medicine, Daegu, Korea \\ ${ }^{9}$ Health Promotion Center, Kyungpook National University Hospital, Daegu, Korea \\ ${ }^{10}$ Center for Genome Science, National Institute of Health, Osong Health Technology Administration complex, Cheongju, Korea
}

Purpose: Pulse wave velocity (PWV) is an indicator of arterial stiffness, and is considered a marker of vascular damage. However, a genome-wide association study analyzing single nucleotide polymorphisms (SNPs) associated with brachial-ankle PWV (baPWV) has not been conducted in healthy populations. We performed this study to identify SNPs associated with baPWV in healthy populations in Korea.

Materials and Methods: Genomic SNPs data for 2,407 individuals from three sites were analyzed as part of the Korean Genomic Epidemiologic Study. Without replication samples, we performed multivariable analysis as a post hoc analysis to verify the findings in site adjusted analysis. Healthy subjects aged between 40 and 70 years without self-reported history or diagnosis of hypertension, diabetes, hyperlipidemia, heart disease, cerebrovascular disease and cancer were included. We excluded subjects with a creatinine level $>1.4 \mathrm{mg} / \mathrm{dL}$ (men) and $1.2 \mathrm{mg} / \mathrm{dL}$ (women).

Results: In the site-adjusted association analysis, significant associations $\left(P<5 \times 10^{-8}\right)$ with baPWV were detected for only 5 SNPs with low minor allele frequency. In multivariable analysis adjusted by age, sex, height, body mass index, mean arterial pressure, site, smoking, alcohol, and exercise, $11 \mathrm{SNPs}$ were found to be associated $\left(P<5 \times 10^{-8}\right)$ with baPWV. The 5 SNPs $\left(P<5 \times 10^{-8}\right)$ linked to three genes (OPCML, PRR35 and $\left.R A B 40 C\right)$ were common between site-adjusted analysis and multivariable analysis. However, meta-analysis of the result from three sites for the 11 SNPs showed no significant associations. Conclusion: Using the recent standard for genome-wide association study, we did not find any evidence of significant association signals with baPWV.

Key words: Genome-wide association study, Association, Nucleotides, Pulse.

Received: 6 February 2017, Revised: 15 March 2017, Accepted: 31 March 2017, Published: 30 June 2017

${ }^{*}$ Corresponding author: Jinho Shin, M.D., Ph.D.

Department of Internal Medicine, Hanyang University College of Medicine, 222 Wansimni-ro, Seongdong-gu, Seoul 04763, Korea.

Tel: +82-2-2290-8308, Fax: +82-2-2299-0278, E-mail: jhs2003@hanyang.ac.kr

Conflict of interest: The authors declare that they do not have any conflicts of interest.

(c) This is an open-access article distributed under the terms of the Creative Commons Attribution Non-Commercial License (http://creativecommons.org/licenses/by-nc/4.0/) which permits unrestricted non-commercial use, distribution, and reproduction in any medium, provided the original work is properly cited.

(c) Copyright 2017 by the Korean Society of Medical Genetics and Genomics

www.e-kjgm.org 


\section{Introduction}

Cardiovascular disease is a substantial cause of morbidity and mortality worldwide and aging is an independent risk factor for cardiovascular disease. Aging of the arterial system is accompanied by structural changes including fragmentation and degeneration of elastin, an increase in collagen, thickening of the arterial walls, and progressive dilatation of arteries. These changes result in a gradual stiffening of the vasculature.

Arterial stiffness is a general term that refers to the elasticity of arteries, reflecting the state of vessel wall diastolic function and buffering capacity. Arterial stiffness occurs as a consequence of age and arteriosclerosis. Age-related stiffness occurs when elastic fibers within the arterial wall (elastin) begin to fray due to mechanical stress. The two leading causes of death in the developed world, myocardial infarction and stroke, are both a direct consequence of atherosclerosis. Atherosclerosis progression can be clinically evaluated by examinations that are able to identify its different aspects [1].

Increased arterial stiffness is associated with an increased risk of cardiovascular events. Pulse wave velocity (PWV) is an indicator of arterial stiffness [2,3], and a marker of vascular damage [4-6]. Thus, in the review of 2013 Korean Society of Hypertension guidelines for the management of hypertension, PWV was also advanced as a method of detecting subclinical organ damage [7]. PWV is the velocity of propagation of forward and backward pressure waves between two points on the arterial tree [8], generally between the carotid and femoral arteries, and represents the mean rigidity between the two points. Carotidfemoral PWV (cfPWV) measurement is one of the most popular techniques for estimating the level of arterial stiffness; however, accurate measurement of cfPWV levels requires specialized training, and the procedure can be cumbersome.

Brachial-ankle PWV (baPWV) measurement, which is easy to perform, has recently become available in clinical practice [9]. For example, the well-known study by Kawai et al. [10,11] used baPWV as a measure of arterial stiffness. Its validity and utility have been proven in various clinical settings [12-17] and in meta-analyses [18,19], and many studies have investigated its clinical significance $[9,20]$. baPWV can easily be calculated using pressure cuffs on the brachial and tibial arteries. It reflects the stiffness of central to middle-sized arteries, and can be applied as a first line tool. Additionally, baPWV is closely correlated with cfPWV.

Recent genetic studies have identified specific genes that contribute to arterial stiffening, and genome-wide linkage anal- yses have revealed genetic polymorphisms associated with arterial stiffness. However, PWV is affected by various factors other than genetic background including blood pressure, diabetes, hypercholesterolemia, and renal function. Genetic association studies with statistical adjustment for these covariates have not been reported. We performed this study to identify the single nucleotide polymorphisms (SNPs) associated with baPWV in three rural areas in Korea.

\section{Materials and Methods}

\section{Study population}

The study subjects were selected from the cohort of an ongoing population-based study designated as the Korean Genome and Epidemiology Study (KoGES). Participants were recruited from among residents aged 38-89 years from three rural areas: Yangpyeong in Gyeonggi-do province, Goryeong in Gyeongsangbuk-do province, and Namwon in Jeollabuk-do province. In total, 8,702 males and females were recruited for the baseline study between 2004 and 2008. The study was approved by the Institutional Review Board (IRB) of the Korea National Institute of Health and all study subjects gave written informed consent before participation (IRB No. HYUH IRB 2005-15, HYUH IRB 2006-32 and HYUH IRB 2007-04).

\section{General characteristics and anthropometric measurements}

All participants were interviewed by trained interviewers, and general information (such as age, sex, smoking status, alcohol consumption, past medical history) was collected using a structured questionnaire. The criterion for regular exercise was $\geq 3$ times per week and $\geq 30$ minutes per session. Smoking status was classified as non-smoker, past smoker, or current smoker. Alcohol consumption was classified as non-drinker, past drinker, or current drinker.

In addition, $10 \mathrm{~mL}$ samples of fasting venous blood were collected in plain tubes. Sera were isolated by centrifugation at $3,000 \mathrm{rpm}$ for 10 minutes at $4^{\circ} \mathrm{C}$ and were stored in liquid nitrogen. Biochemical tests including fasting serum glucose, fasting serum insulin, total cholesterol, triglyceride (TG), high density lipoprotein-cholesterol (HDL-cholesterol), and others were conducted.

The anthropometric characteristics (such as height, weight, waist circumference, blood pressure) were measured according to standardized protocols. Waist circumference was measured half way between the lowest rib margin and the iliac crest. For measurement of blood pressure, subjects had been sitting for at 
least 5 minutes. Systolic blood pressure (SBP) and diastolic blood pressure (DBP) were measured with a standard mercury sphygmomanometer using the first and fifth Korotkoff sounds, to the nearest $2 \mathrm{mmHg}$. If two systolic or DBP readings were more than $5 \mathrm{mmHg}$ apart, an additional measurement was performed, and the mean of the last two measurements was used for subsequent analyses.

From the total of 8,702 subjects that were identified, we excluded subjects that had self-reported disease histories and were receiving drug-treatment for hypertension, type 2 diabetes mellitus, hyperlipidemia, heart disease, cerebrovascular diseases, and cancer. We also excluded subjects who were diagnosed with hypertension by supine position blood pressure measurements (SBP $\geq 140 \mathrm{mmHg}$ and/or DBP $\geq 90 \mathrm{mmHg}$ ), and type 2 diabetes by fasting plasma glucose level (Glu $\geq 126 \mathrm{mg} / \mathrm{dL}$ ). Finally, 4,034 healthy subjects that did not have a history or characteristics of any of the six diseases were selected for SNP genotyping, and 3,667 of these subjects fulfilled the quality control specifications included in the study.

The following participants were excluded: participants who were $\leq 40$ or $\geq 70$ years of age; those with missing height or weight values; with total cholesterol $\geq 250 \mathrm{mg} / \mathrm{dL}$ or creatinine $>1.4 \mathrm{mg} / \mathrm{dL}$ for men and creatinine $>1.2 \mathrm{mg} / \mathrm{dL}$ for women; subjects with missing alcohol consumption or smoking histories, or unmeasured right brachial SBP or pulse rate $>100$ beats/min during arterial stiffness measurement; and those with missing exercise variable values in the structured questionnaire. Based on these exclusion criteria, 2,407 of the 3,667 individuals were included in the study.

\section{Genotyping and quality control}

In total, 4,034 KoGES Cardiovascular Disease Association study samples were genotyped from the three rural areas using $\mathrm{Hu}$ manOmni1 Quad v1.0 (Illumina, San Diego, CA, USA). Genotypes were called using Bead Studio (IIlumina, San Diego, CA, USA). Samples that exhibited the following properties were excluded: low genotyping calls $<98 \%(n=185)$, excessive heterozygosity $(n=61)$, sex inconsistency $(n=18)$, and cryptic relatedness $(n=4)$. Markers with high missing gene call rates ( $>5 \%)$, rare minor allele frequency (MAF; $<0.01)$, or significant deviation from the Hardy-Weinberg equilibrium $\left(P<1 \times 10^{-6}\right)$ were also excluded. To detect the population substructure, we performed multidimensional scaling and principal component analyses using PLINK software (PLINK v1.07; Purcell Lab, New York, NY, USA) and R programming language (R Foundation for Statistical Computing, Vienna, Austria). Dimensions were calculated on the basis of identity-by-descent pair-wise distances between all individuals. The degree of relation was determined in individuals $(n=99)$ whose estimated identity-by-state values were high $(>0.80)$. After these quality control steps, 3,667 samples were selected. Markers with high missing gene call rates ( $>5 \%$ ), rare MAF $(<0.01)$, and significant deviations from the Hardy-Weinberg equilibrium $\left(P<1 \times 10^{-6}\right)$ were excluded, leaving a total of 723,056 markers for examination in 3,667 individuals.

\section{Measurement of brachial-ankle pulse wave velocity and right brachial systolic pressure}

Arterial tonometry was conducted to assess arterial stiffness in all the cohort participants during their examinations. Two primary tonometry phenotypes were analyzed: left and right PWV. PWV was measured using the VP-2000. Tonometry was performed in a supine position after 5 minutes of rest. Arterial tonometry was obtained with simultaneous ECG recording.

\section{Statistical analysis}

General characteristics were analyzed using SAS 9.2. (SAS Institute, Cary, NC, USA) Measurement data were expressed as means \pm standard deviations, and count data as percentages. Genetic analysis was conducted using PLINK software (PLINK v1.07; Purcell Lab, New York, NY, USA). In the genetic analysis, standardized multivariable-adjusted PWV and tonometry residuals were generated as described above. Mean arterial pressure (MAP) was calculated from the systolic and DBP. The dependent variable, mean baPWV, was transformed to a normal distribution using reciprocal transformation methods, and the covariates adjusted were age, sex, height, body mass index (BMI), MAP, site information, smoking status, alcohol consumption, and exercise. The numerical covariates were also transformed to form as normal a distribution as possible. A $P$-value $<5 \times 10^{-8}$ was regarded as statistically significant.

\section{Results}

\section{General characteristics}

The mean age of the 2,407 individuals was 56.6 years; $36.1 \%$ of the participants were male and the mean BMI was $24.0 \pm 3.0$ $\mathrm{kg} / \mathrm{m}^{2}$. Subjects that smoked accounted for $16.2 \%$ of the total study population. Alcohol consumption was noted in $47.3 \%$ of the study subjects. Of the total study population, 30.2\% exercised regularly. Casual SBP was $115.1 \pm 11.1 \mathrm{mmHg}$, casual MAP was $88.6 \pm 7.9 \mathrm{mmHg}$ and casual DBP was $75.4 \pm 7.5 \mathrm{mmHg}$. Blood sample analysis indicated that the mean uric acid level was 
$4.7 \pm 1.3 \mathrm{mg} / \mathrm{dL}$, total cholesterol was $190.6 \pm 29.4 \mathrm{mg} / \mathrm{dL}, \mathrm{HDL}-$ cholesterol was $45.0 \pm 10.7 \mathrm{mg} / \mathrm{dL}$, TGs were $133.7 \pm 79.1 \mathrm{mg} / \mathrm{dL}$, glucose was $93.8 \pm 9.6 \mathrm{mg} / \mathrm{dL}$, and creatinine was $0.9 \pm 0.1 \mathrm{mg} /$ $\mathrm{dL}$. Right brachial pulse rates were $64.8 \pm 9.3$ beats $/ \mathrm{min}$. In the baPWV, the right baPWV was $1,390.8 \pm 228.5 \mathrm{~cm} / \mathrm{s}$, the left was $1,403.0 \pm 227.2 \mathrm{~cm} / \mathrm{s}$, and the mean baPWV was $1,396.9 \pm 224.4$ $\mathrm{cm} / \mathrm{s}$. Table 1 also shows the characteristics of these variables at each study site, including the observation that mean baPWV differed across the study sites (Yangpyeong: 1,387.0 $220.5 \mathrm{~cm} /$ s, Namwon: 1,421.2 $\pm 232.3 \mathrm{~cm} / \mathrm{s}$, and Goryeong: 1,388.8 \pm 221.2 $\mathrm{cm} / \mathrm{s} ; P=0.0049)$.

\section{Site information adjusted association}

The arterial stiffness phenotype, mean baPWV, was used as an indicator for the site information adjusted association analysis. After applying the exclusion criteria, 723,056 SNPs were included in the analysis. The separate analysis adjusted by site information is shown in Table 2 and Fig 1. Nine SNPs had $P$ values $<10^{-6}$ for the association with baPWV. Of these, five SNPs (rs476840, rs11607110, rs1010761, rs11643412, and rs11648607)

Table 1. Characteristics of the general study population

\begin{tabular}{|c|c|c|c|c|}
\hline & Yangpyeong $(n=957)$ & Namwon $(n=654)$ & Goryeong $(n=796)$ & Total $(n=2407)$ \\
\hline Age (yr) & $55.0 \pm 8.7$ & $56.3 \pm 8.1$ & $57.9 \pm 7.6$ & $56.6 \pm 8.3$ \\
\hline Male/female (n) & $347 / 610(36.3 / 63.7)$ & $245 / 409(37.5 / 62.5)$ & $276 / 520(34.7 / 65.3)$ & 868/1,539 (36.1/63.9) \\
\hline BMI $\left(\mathrm{kg} / \mathrm{m}^{2}\right)$ & $24.3 \pm 2.9$ & $23.7 \pm 2.8$ & $24.0 \pm 3.0$ & $24.0 \pm 3.0$ \\
\hline Smoking (n) & $143(14.9)$ & $127(19.4)$ & 121 (15.2) & 391 (16.2) \\
\hline Alcohol (n) & $485(50.7)$ & $290(44.3)$ & $364(45.7)$ & $1139(47.3)$ \\
\hline Exercise (n) & $314(32.8)$ & $196(30.0)$ & $217(27.3)$ & 727 (30.2) \\
\hline $\mathrm{SBP}(\mathrm{mmHg})$ & $114.4 \pm 10.6$ & $115.7 \pm 11.1$ & $115.4 \pm 11.7$ & $115.1 \pm 11.1$ \\
\hline $\operatorname{MAP}(\mathrm{mmHg})$ & $88.8 \pm 7.6$ & $87.8 \pm 8.2$ & $89.0 \pm 8.0$ & $88.6 \pm 7.9$ \\
\hline $\mathrm{DBP}(\mathrm{mmHg})$ & $76.0 \pm 7.2$ & $73.8 \pm 7.9$ & $75.9 \pm 7.3$ & $75.4 \pm 7.5$ \\
\hline Uric acid (mg/dL) & $4.7 \pm 1.2$ & $4.9 \pm 1.3$ & $4.6 \pm 1.3$ & $4.7 \pm 1.3$ \\
\hline T-cholesterol (mg/dL) & $189.4 \pm 29.8$ & $194.4 \pm 28.4$ & $188.9 \pm 29.3$ & $190.6 \pm 29.4$ \\
\hline HDL-cholesterol (mg/dL) & $45.2 \pm 10.8$ & $43.8 \pm 10.1$ & $45.7 \pm 10.8$ & $45.0 \pm 10.7$ \\
\hline $\mathrm{TG}(\mathrm{mg} / \mathrm{dL})$ & $134.5 \pm 78.9$ & $130.3 \pm 77.5$ & $135.5 \pm 80.7$ & $133.7 \pm 79.1$ \\
\hline $\mathrm{FBG}(\mathrm{mg} / \mathrm{dL})$ & $95.4 \pm 9.3$ & $91.2 \pm 9.2$ & $93.9 \pm 9.7$ & $93.8 \pm 9.6$ \\
\hline Creatinine $(\mathrm{mg} / \mathrm{dL})$ & $0.9 \pm 0.1$ & $0.9 \pm 0.1$ & $0.9 \pm 0.1$ & $0.9 \pm 0.1$ \\
\hline Right brachial pulse rate (beats/min) & $64.4 \pm 9.5$ & $66.3 \pm 9.5$ & $64.1 \pm 8.7$ & $64.8 \pm 9.3$ \\
\hline Right baPWV (cm/sec) & $1,381.0 \pm 225.7$ & $1,416.3 \pm 234.5$ & $1,381.6 \pm 225.6$ & $1,390.8 \pm 228.5$ \\
\hline Left baPWV (cm/sec) & $1,393.0 \pm 221.0$ & $1,426.2 \pm 235.9$ & $1,396.0 \pm 226.1$ & $1,403.0 \pm 227.2$ \\
\hline Mean baPWV (cm/sec) & $1,387.0 \pm 220.5$ & $1,421.2 \pm 232.3$ & $1,388.8 \pm 221.2$ & $1,396.9 \pm 224.4$ \\
\hline
\end{tabular}

Values are presented as mean \pm standard deviation or number (\%).

BMI, body mass index; SBP, systolic blood pressure; MAP, mean arterial pressure; DBP, diastolic blood pressure; T-cholesterol, total-cholesterol; HDLcholesterol, high-density lipoprotein-cholesterol; TG, Triglyceride; FBG, fasting blood glucose; baPWV, brachial ankle pulse wave velocity.

Table 2. Description of SNPs in the association study with adjustment for site information $\left(P<10^{-6}\right)$

\begin{tabular}{lccccccccccc}
\multicolumn{1}{c}{ SNP } & Chromosome & Position & Locus & Gene & Location Minor allele & MAF & RAF & $\beta$ & SE & $P^{\text {a }}$ \\
\hline rs2645290 & 1 & 119273431 & $1 p 11.1$ & TBX15 & Intron & A & 0.1586 & 0.159 & -0.1714 & 0.03462 & $7.82 \mathrm{E}-07$ \\
rs1766787 & 1 & 119254281 & $1 p 11.1$ & TBX15 & Intron & C & 0.1588 & 0.159 & -0.1714 & 0.03463 & $7.9 \mathrm{E}-07$ \\
\hline rs2148150 & 1 & 119373580 & - & - & - & A & 0.1521 & 0.152 & -0.167 & 0.03381 & $8.45 \mathrm{E}-07$ \\
rs476840 & 11 & 132568537 & $11 q 25$ & OPCML & Intron & C & 0.02168 & 0.0217 & 0.6169 & 0.1011 & $1.21 \mathrm{E}-09$ \\
rs11607110 & 11 & 132576733 & - & - & - & A & 0.02154 & 0.0215 & 0.614 & 0.1006 & $1.22 \mathrm{E}-09$ \\
rs2073350 & 14 & 23614088 & $14 q 11.2$ & CPNE6 & Intron & A & 0.4138 & 0.414 & 0.1066 & 0.02089 & $3.57 \mathrm{E}-07$ \\
rs1010761 & 16 & 554822 & $16 p 13.3$ & PRR35 & Intron & G & 0.0251 & 0.0251 & 0.7008 & 0.1148 & $1.19 \mathrm{E}-09$ \\
rs11643412 & 16 & 605324 & $16 p 13.3$ & RAB40C & Intron & G & 0.03218 & 0.0322 & 0.5031 & 0.09122 & $3.86 \mathrm{E}-08$ \\
rs11648607 & 16 & 611246 & $16 p 13.3$ & RAB40C & Intron & A & 0.03178 & 0.0318 & 0.4892 & 0.08881 & $4.02 \mathrm{E}-08$ \\
\hline
\end{tabular}

asite information was adjusted.

SNP, single nucleotide polymorphism; MAF, minor allele frequency; RAF, Risk allele frequency; $\beta$, regression coefficient; SE, standard error. 
had $P$-values $<5 \times 10^{-8}$ and were linked to three genes $(O P C M L$, PRR35, RAB40C). However, the MAFs were all low, i.e., between

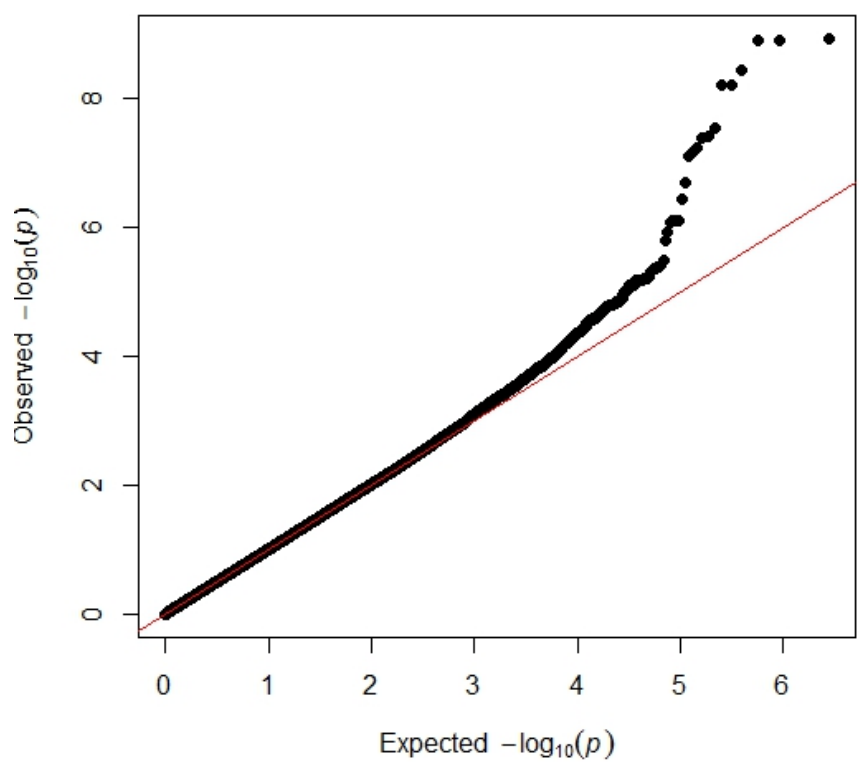

Fig. 1. $Q-Q$ plot showing the genome-wide $P$-values for site information adjusted associations. Lambda $\mathrm{GC}=0.996$. The plot shows $P$ values for all the single nucleotide polymorphisms that were analyzed. Note that the circles in the right half of the plot are significantly above the line. A QQ-plot of these $P$-values was used to see if the very low $P$ values differed from the expected distribution (a uniform distribution). $\mathrm{GC}$, genomic control; $\mathrm{Q}-\mathrm{Q}$, quantile-quantile.
0.01 and 0.05 .

\section{Multivariable adjusted association}

The variations in age, sex, height, BMI, MAP, site information, smoking status, alcohol consumption, and exercise variables were adjusted for the arterial stiffness phenotype analysis. Covariate analysis included adjustment factors such as age, sex, and multiple variables. After adjusting for multiple confounding factors, it appeared that 18 SNPs had $P$ values $<10^{-6}$ for the association with baPWV. Of these, 11 SNPs (rs11993165, rs7121368, rs476840, rs11607110, rs10152599, rs2279496, rs7172171, rs16955052, rs1010761, rs11643412, and rs 11648607) had $P$-values $<5 \times 10^{-8}$ and were linked to five genes (PDGFRL, OPCML, APBA2, PRR35 and RAB4OC) as shown in Table 3 and Figs. 2 and 3.

From the above results, five SNPs were common between site information adjusted association and multivariable adjusted association, with a $P$-value $<5 \times 10^{-8}$, and were linked to three genes (OPCML, PRR35, and RAB4OC).

$\mathrm{OPCML}$, the opioid-binding protein/cell adhesion molecule, is a protein that is encoded by the OPCML gene in humans [21,22]. This gene encodes a member of the IgLON subfamily in the immunoglobulin superfamily of proteins. The encoded pre-protein is proteolytically processed to generate the mature protein.

Table 3. Description of SNPs in the multivariable adjusted association study $\left(P<10^{-6}\right)$

\begin{tabular}{|c|c|c|c|c|c|c|c|c|c|c|c|}
\hline SNP & Chromosome & Position & Locus & Gene & Location & Minor allele & MAF & RAF & $\beta$ & SE & $P^{a}$ \\
\hline rs2046590 & 2 & 186604548 & - & - & - & A & 0.0629 & 0.0629 & 0.2366 & 0.0461 & 3.06E-07 \\
\hline rs1038587 & 2 & 186614035 & $2 q 32.1$ & LINC01473 & Intron & A & 0.0621 & 0.0621 & 0.2727 & 0.0554 & $9.26 \mathrm{E}-07$ \\
\hline rs11993165 & 8 & 17527398 & 8p22-p21.3 & PDGFRL & Intron & A & 0.0405 & 0.0405 & 0.3015 & 0.0538 & 2.26E-08 \\
\hline rs7844026 & 8 & 93622913 & - & - & - & A & 0.0409 & 0.0409 & 0.4011 & 0.0777 & 2.67E-07 \\
\hline rs510960 & 11 & 132565481 & $11 q 25$ & OPCML & Intron & G & 0.0307 & 0.0307 & 0.3524 & 0.0681 & 2.51E-07 \\
\hline rs7121368 & 11 & 132567618 & $11 q 25$ & OPCML & Intron & A & 0.042 & 0.042 & 0.4712 & 0.0784 & 2.12E-09 \\
\hline rs476840 & 11 & 132568537 & $11 q 25$ & $O P C M L$ & Intron & C & 0.0217 & 0.0217 & 0.6678 & 0.0797 & $9.31 \mathrm{E}-17$ \\
\hline rs11607110 & 11 & 132576733 & - & - & - & A & 0.0215 & 0.0215 & 0.6646 & 0.0794 & 9.37E-17 \\
\hline rs2900264 & 12 & 12357203 & - & - & - & A & 0.0942 & 0.0942 & 0.1749 & 0.0346 & 4.61E-07 \\
\hline rs10152599 & 15 & 27149506 & $15 q 11-q 12$ & APBA2 & Intron & A & 0.0296 & 0.0296 & 0.3864 & 0.0681 & $1.54 \mathrm{E}-08$ \\
\hline rs2279496 & 15 & 27155608 & $15 q 11-q 12$ & APBA2 & Intron & G & 0.0296 & 0.0296 & 0.3866 & 0.0681 & $1.50 \mathrm{E}-08$ \\
\hline rs7172171 & 15 & 27157470 & $15 q 11-q 12$ & APBA2 & Intron & A & 0.0296 & 0.0296 & 0.3865 & 0.068 & $1.51 \mathrm{E}-08$ \\
\hline rs16955052 & 15 & 27167415 & $15 q 11-q 12$ & APBA2 & Intron & G & 0.03 & 0.03 & 0.3888 & 0.0685 & $1.52 \mathrm{E}-08$ \\
\hline rs1010761 & 16 & 554822 & 16p13.3 & PRR35 & Intron & G & 0.0251 & 0.0251 & 0.7583 & 0.0906 & $9.30 \mathrm{E}-17$ \\
\hline rs11643412 & 16 & 605324 & 16p13.3 & $R A B 40 C$ & Intron & G & 0.0322 & 0.0322 & 0.5298 & 0.0723 & $3.11 \mathrm{E}-13$ \\
\hline rs 11648607 & 16 & 611246 & 16p13.3 & RAB4OC & Intron & A & 0.0318 & 0.0318 & 0.5153 & 0.0703 & $3.22 \mathrm{E}-13$ \\
\hline rs1672228 & 18 & 47182761 & $18 \mathrm{q} 21.2$ & LOC100287225 & Intron & A & 0.1763 & 0.176 & 0.1297 & 0.0253 & 3.06E-07 \\
\hline rs821195 & 18 & 47190580 & $18 \mathrm{q} 21.2$ & LOC100287225 & Intron & A & 0.1668 & 0.167 & 0.1314 & 0.026 & 4.46E-07 \\
\hline
\end{tabular}

${ }^{a} \mathrm{Age}$, sex, height, body mass index, mean arterial pressure, site information, smoking status, alcohol consumption and exercise variables were adjusted. SNP, single nucleotide polymorphism; MAF, minor allele frequency; RAF, Risk allele frequency; $\beta$, regression coefficient; SE, standard error. 


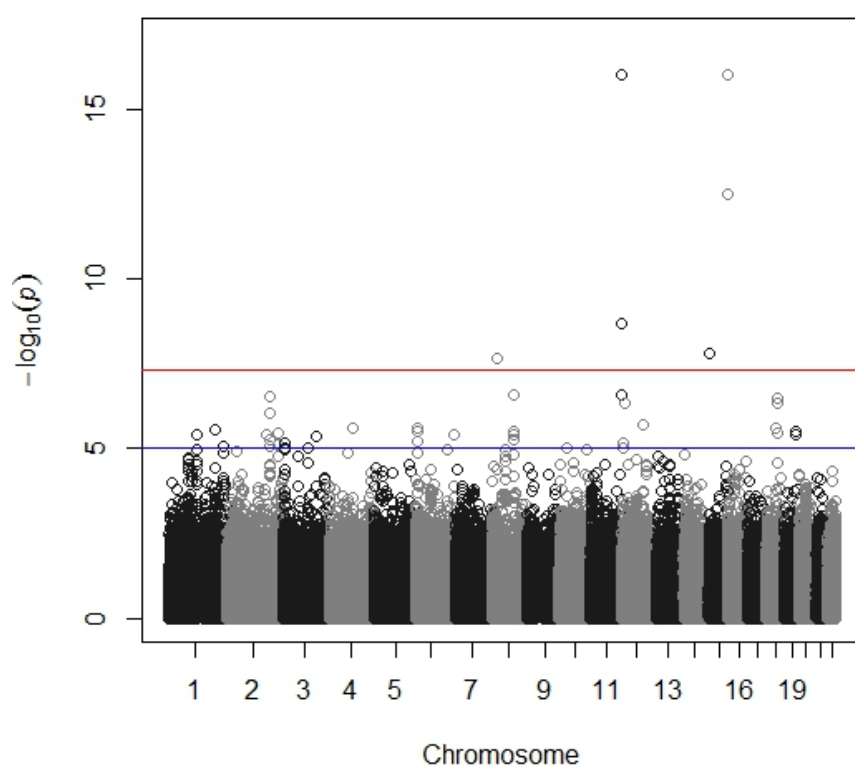

Fig. 2. Manhattan plot showing genome-wide $P$-values of multiple associations. The minimum $P$-values under the genetic models were obtained by linear regression analysis with adjustment for age, sex, height, body mass index, mean arterial pressure, site information, smoking status, alcohol consumption, and exercise. The y axis shows the $-\log _{10} P$-values of 723,056 single nucleotide polymorphisms, and the $x$ axis shows their chromosomal positions. The horizontal red and blue lines represent the thresholds of $P=3.47 \times 10^{-8}$ for Bonferroni's significance and $P=1 \times 10^{-5}$ for selecting the SNPs for replication, respectively.

This protein is localized in the plasma membrane and may have an accessory role in opioid receptor function. This gene has an ortholog in rats and bovines. The opioid binding-cell adhesion molecule encoded by the rat gene binds opioid alkaloids in the presence of acidic lipids, exhibits selectivity for mu ligands, and acts as a glucose-6-phosphate isomerase-anchored protein. Since the encoded protein is highly conserved across species during evolution, it may have a fundamental role in mammalian systems. Two transcript variants encoding different isoforms have been found for this gene [22]. Alternative splicing results in multiple transcript variants, at least one of which encodes an isoform that is proteolytically processed (provided by RefSeq, Jan 2016). PRR35 or proline rich 35 , is a protein that is encoded by the PRR35 gene in humans [23]. RAB40C is a member of the renin-angiotensin system oncogene family, but its specific role and mechanism has not been elucidated in the current study.

\section{Multivariable adjusted association analyses for three study sites}

For the 11 SNPs, multivariable analyses were preformed separately for three study sites as shown in Table 4, and the effect sizes for all 11 SNPs were extremely large only in Yangpyeong.

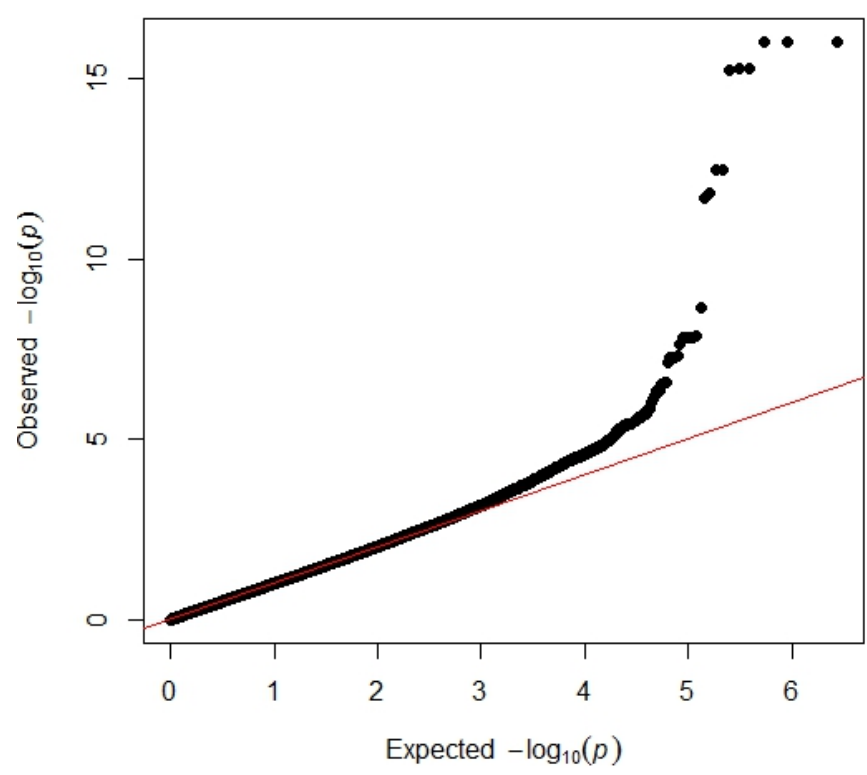

Fig. 3. $Q-Q$ plot showing genome-wide $P$-values in the multiple associations. Lambda $\mathrm{GC}=0.995$. The plot shows $P$-values for all the single nucleotide polymorphisms that were analyzed. Note that the circles in the right half of the plot are significantly above the line. A QQ-plot of these $P$-values was used to see if the very low $P$-values differed from the expected distribution (a uniform distribution).

\section{Meta-analysis}

For the 11 SNPs, the results from the three study sites were meta-analyzed. Among the 11 SNPs, none was associated with baPWV with $P$-values $<5 \times 10^{-6}$ as shown in Table 5 .

\section{Discussion}

\section{Main findings}

The main finding of the present study, which was performed in a healthy and normal general population, was that 5 SNPs were common between site information adjusted association and the multivariable adjusted association analyses with regard to baPWV. The multiple adjusted model was provided as a post hoc analysis to verify the findings of the site information adjusted association analysis. However, such associations with low MAF seemed to be noise signals because the multivariable analyses performed for the three individual study sites and the corresponding meta-analyses showed no significant SNPs associated with baPWV. From the statistical viewpoint, these were considered as negative findings.

\section{Previous genome-wide association studies on arterial stiffness}

Previous investigations of the association between arterial stiffness and SNPs using genome-wide association studies 
Table 4. Description of SNPs in the multivariable adjusted association at each study site

\begin{tabular}{|c|c|c|c|c|c|c|c|c|c|}
\hline Chromosome & SNP & Position & A1 & $\beta$ & SE & L95 & U95 & STAT & $P^{\mathrm{a}}$ \\
\hline \multicolumn{10}{|l|}{ In Yangpyeong } \\
\hline 8 & rs11993165 & 17527398 & A & 0.608 & 0.07685 & 0.4574 & 0.7586 & 7.912 & 7.06E-15 \\
\hline 11 & rs7121368 & 132567618 & A & 0.4816 & 0.07932 & 0.3261 & 0.6371 & 6.072 & 1.83E-09 \\
\hline 11 & rs476840 & 132568537 & C & 0.7149 & 0.08424 & 0.5498 & 0.88 & 8.487 & 8.13E-17 \\
\hline 11 & rs11607110 & 132576733 & A & 0.7149 & 0.08424 & 0.5498 & 0.88 & 8.487 & 8.13E-17 \\
\hline 15 & rs10152599 & 27149506 & A & 0.3873 & 0.06787 & 0.2543 & 0.5203 & 5.707 & $1.54 \mathrm{E}-08$ \\
\hline 15 & rs2279496 & 27155608 & G & 0.3873 & 0.0679 & 0.2542 & 0.5204 & 5.704 & 1.57E-08 \\
\hline 15 & rs7172171 & 27157470 & A & 0.3873 & 0.06787 & 0.2543 & 0.5203 & 5.707 & $1.54 \mathrm{E}-08$ \\
\hline 15 & rs16955052 & 27167415 & G & 0.3873 & 0.06787 & 0.2543 & 0.5203 & 5.707 & $1.54 \mathrm{E}-08$ \\
\hline 16 & rs1010761 & 554822 & G & 0.7414 & 0.08737 & 0.5701 & 0.9126 & 8.486 & $8.21 \mathrm{E}-17$ \\
\hline 16 & rs11643412 & 605324 & G & 0.5093 & 0.06738 & 0.3772 & 0.6414 & 7.559 & 9.59E-14 \\
\hline 16 & rs11648607 & 611246 & A & 0.502 & 0.06636 & 0.3719 & 0.632 & 7.565 & $9.21 \mathrm{E}-14$ \\
\hline \multicolumn{10}{|l|}{ In Namwon } \\
\hline 8 & rs11993165 & 17527398 & A & -0.01274 & 0.0324 & -0.07624 & 0.05077 & -0.3931 & $6.94 \mathrm{E}-01$ \\
\hline 11 & rs7121368 & 132567618 & A & 0.009672 & 0.03205 & -0.05315 & 0.07249 & 0.3018 & 7.63E-01 \\
\hline 11 & rs476840 & 132568537 & C & -0.004828 & 0.03207 & -0.06768 & 0.05802 & -0.1506 & $8.80 \mathrm{E}-01$ \\
\hline 11 & rs11607110 & 132576733 & A & -0.004828 & 0.03207 & -0.06768 & 0.05802 & -0.1506 & 8.80E-01 \\
\hline 15 & rs10152599 & 27149506 & A & -0.003536 & 0.03217 & -0.06658 & 0.05951 & -0.1099 & $9.13 \mathrm{E}-01$ \\
\hline 15 & rs2279496 & 27155608 & G & -0.003491 & 0.03215 & -0.06651 & 0.05952 & -0.1086 & $9.14 \mathrm{E}-01$ \\
\hline 15 & rs7172171 & 27157470 & A & -0.003491 & 0.03215 & -0.06651 & 0.05952 & -0.1086 & $9.14 \mathrm{E}-01$ \\
\hline 15 & rs16955052 & 27167415 & G & -0.003491 & 0.03215 & -0.06651 & 0.05952 & -0.1086 & $9.14 \mathrm{E}-01$ \\
\hline 16 & rs1010761 & 554822 & G & 0.03157 & 0.03205 & -0.03125 & 0.09439 & 0.9849 & 3.25E-01 \\
\hline 16 & rs11643412 & 605324 & G & 0.02053 & 0.0323 & -0.04277 & 0.08384 & 0.6357 & 5.25E-01 \\
\hline 16 & rs11648607 & 611246 & A & -0.01115 & 0.03223 & -0.07433 & 0.05202 & -0.346 & 7.30E-01 \\
\hline \multicolumn{10}{|l|}{ In Goryeong } \\
\hline 8 & rs11993165 & 17527398 & A & -0.05761 & 0.02744 & -0.1114 & -0.00382 & -2.099 & $3.61 \mathrm{E}-02$ \\
\hline 11 & rs7121368 & 132567618 & A & -0.0159 & 0.02746 & -0.06971 & 0.03791 & -0.5792 & 5.63E-01 \\
\hline 11 & rs476840 & 132568537 & C & 0.004207 & 0.02744 & -0.04958 & 0.058 & 0.1533 & 8.78E-01 \\
\hline 11 & rs11607110 & 132576733 & A & -0.0009364 & 0.02745 & -0.05474 & 0.05287 & -0.03411 & $9.73 \mathrm{E}-01$ \\
\hline 15 & rs10152599 & 27149506 & A & -0.01507 & 0.02743 & -0.06883 & 0.0387 & -0.5492 & 5.83E-01 \\
\hline 15 & rs2279496 & 27155608 & G & -0.01488 & 0.02741 & -0.0686 & 0.03883 & -0.543 & 5.87E-01 \\
\hline 15 & rs7172171 & 27157470 & A & -0.01523 & 0.02742 & -0.06896 & 0.03851 & -0.5553 & 5.79E-01 \\
\hline 15 & rs16955052 & 27167415 & G & -0.02166 & 0.0274 & -0.07536 & 0.03204 & -0.7904 & 4.30E-01 \\
\hline 16 & rs 1010761 & 554822 & G & -0.009659 & 0.02751 & -0.06359 & 0.04427 & -0.351 & 7.26E-01 \\
\hline 16 & rs11643412 & 605324 & G & 0.005239 & 0.02761 & -0.04888 & 0.05935 & 0.1897 & 8.50E-01 \\
\hline 16 & rs11648607 & 611246 & A & -0.002022 & 0.02765 & -0.05622 & 0.05217 & -0.07313 & $9.42 \mathrm{E}-01$ \\
\hline
\end{tabular}

${ }^{a} \mathrm{Age}$, sex, height, body mass index, mean arterial pressure, smoking status, alcohol consumption and exercise variables were adjusted.

SNP, single nucleotide polymorphism; A1, first allele code; $\beta$, regression coefficient; SE, standard error; L95, lower bound of 95\% confidence interval; U95, upper bound of $95 \%$ confidence interval; STAT, coefficient t-statistic.

(GWAS) involved pulse pressure and PWV. The genes identified in the various studies using pulse pressure, which included the Framingham Heart Study, did not yield consistent findings [24-26]. PWV is currently considered an optimal measure, as recently described in the consensus statement on arterial stiffness [27]. cfPWV is the gold-standard index of arterial stiffness; however, the complexity of measuring cfPWV [28] prevents its use in routine clinical practice. Measurement of baPWV is simple, noninvasive, and more practical for population surveys. However, in the Framingham Offspring Study, as in studies using carotid-brachial PWV, rs 10514688, rs770189, and rs7042864 had significant associations after adjustment for age, sex, age 2 , height, and weight $\left(P<10^{-5}\right)[29]$. However, these SNPs were not associated with baPWV in our study. These results of our study 
Table 5. Meta-analysis results of three study sites

\begin{tabular}{|c|c|c|c|c|c|c|c|c|c|c|c|}
\hline Chromosome & Position & SNP & $\mathrm{A} 1$ & A2 & N & $P$-value ${ }^{a}$ & $P$-value ${ }^{\mathrm{b}}$ & $\beta$ & $\beta(\mathrm{R})$ & $Q$ & $1^{2}$ \\
\hline 8 & 17527398 & rs11993165 & $A$ & $G$ & 3 & 0.7727 & 0.203 & 0.0058 & 0.1655 & 0.0000 & 97.02 \\
\hline 11 & 132567618 & rs7121368 & A & G & 3 & 0.1907 & 0.1468 & 0.0264 & 0.1386 & 0.0000 & 94.38 \\
\hline 11 & 132568537 & rs476840 & C & A & 3 & 0.03969 & 0.09631 & 0.0416 & 0.2201 & 0.0000 & 97.05 \\
\hline 11 & 132576733 & rs11607110 & A & C & 3 & 0.05497 & 0.1002 & 0.0388 & 0.2184 & 0.0000 & 97.07 \\
\hline 15 & 27149506 & rs10152599 & A & G & 3 & 0.2264 & 0.2093 & 0.0241 & 0.1093 & 0.0000 & 93.63 \\
\hline 15 & 27155608 & rs2279496 & G & A & 3 & 0.2252 & 0.2087 & 0.0242 & 0.1093 & 0.0000 & 93.62 \\
\hline 15 & 27157470 & rs7172171 & A & G & 3 & 0.228 & 0.2094 & 0.024 & 0.1092 & 0.0000 & 93.64 \\
\hline 15 & 27167415 & rs16955052 & G & A & 3 & 0.3013 & 0.2228 & 0.0206 & 0.1072 & 0.0000 & 93.78 \\
\hline 16 & 554822 & rs1010761 & G & A & 3 & 0.01945 & 0.07779 & 0.0474 & 0.2347 & 0.0000 & 97.04 \\
\hline 16 & 605324 & rs11643412 & G & A & 3 & 0.005439 & 0.1258 & 0.0557 & 0.1676 & 0.0000 & 95.99 \\
\hline 16 & 611246 & rs11648607 & A & G & 3 & 0.04409 & 0.1749 & 0.0403 & 0.1529 & 0.0000 & 96.25 \\
\hline
\end{tabular}

${ }^{a}$ Fixed-effects meta-analysis, ${ }^{b}$ random-effects meta-analysis.

SNP, single nucleotide polymorphism; A1, first allele code; $A$ 2, second allele code; $N$, number of valid studies for this SNP; $P$, fixed-effects meta-analysis $P$ value; $\beta$, fixed-effects $\beta$ estimate; $\beta(R)$, random-effects $\beta$ estimate; $Q, P$-value for Cochrane's $Q$ statistic; I, I heterogeneity index (0-100).

may be related to differences in ethnicity, study subjects, and other factors. In addition, recent studies also showed that common genetic variants associated with baPWV could not be identified in cardiovascular patients [30]. Compared with our study, this study was based on cardiovascular patients as subjects and factors such as age, sex, SBP, and DBP were adjusted in the model.

\section{Study population}

Previously arterial stiffness and GWAS studies did not exclude subjects with chronic diseases, but the present study was undertaken in a healthy population to exclude the effect of acquired or environmental influences. McEniery et al. [31] and Giannattasio et al. [32] showed that arterial stiffening is associated with aging, hypertension, diabetes mellitus, and chronic kidney disease. We performed a GWAS for arterial stiffness in Korean individuals aged 40 to 70 years. The study subjects included three rural populations, and the analysis focused on distinct SNPs that regulate a specific, pathophysiologically relevant pathway that can alter downstream arterial properties. Moreover, there is increasing evidence that arterial stiffness and cardiovascular events are closely parallel. To the best of our knowledge, this study is the first GWAS to investigate the association between SNPs and baPWV levels in a healthy and normal general population.

\section{Biological consideration: explanation for the genes and SNPs}

Recently, GWAS have shown associations between measures of arterial stiffness and the loci on different chromosomes (chromosomes 2, 7, 13, and 15). However, the pathophysiologi- cal links between the genetic and physiological components remain largely unknown $[26,33,34]$. In addition, some studies have reported that SNPs can affect arterial stiffness [8]; examples include SNPs in genes such as those encoding the renin-angiotensin-aldosterone system, matrix protein/metalloproteinases, the nitric oxide pathway, $\beta$-adrenergic/endothelin receptors, the inflammatory cascade, and others [35-39]. Compared with the current research, this study did not identify specific and relevant SNPs after accounting for various factors. The specific role and mechanism of the 5 SNPs and 3 genes has not been elucidated in the current research, but may be related to arterial stiffness and needs to be studied further.

\section{Limitations of the present study}

The present study has several limitations. First, with regard to age, the study subjects were a middle-aged cohort from three rural populations; therefore, a study needs to be conducted using a young population cohort. Secondly, the study involved a relatively small range of geographical areas and future studies should be conducted in the broader context of national and international populations. Third, in our study, we found 5 SNPs for 3 genes with a $P$-value less than $5 \times 10^{-8}$ and MAFs about 0.02 . With the current knowledge, the implication of an association by these low or rare MAFs cannot be interpreted properly. Because MAFs below 0.05 are usually excluded in standard GWAS, such SNPs with low MAFs shown in our study should not be regarded as an evidence of significant association and further studies for these low or rare MAFs are needed to properly interpret our results [40].

In conclusion, employing the recent standard applied to 
GWAS and due to the lack of replication analysis in the present study, we found no evidence of a significant association signal for baPWV.

\section{References}

1. Takahara M, Katakami N, Osonoi T, Saitou M, Sakamoto F, Matsuoka TA, et al. Different impacts of cardiovascular risk factors on arterial stiffness versus arterial wall thickness in Japanese patients with type 2 Diabetes Mellitus. J Atheroscler Thromb 2015;22:971-80.

2. Lehmann ED. Clinical value of aortic pulse-wave velocity measurement. Lancet 1999;354:528-9.

3. Asmar R, Benetos A, Topouchian J, Laurent P, Pannier B, Brisac AM, et al. Assessment of arterial distensibility by automatic pulse wave velocity measurement. Validation and clinical application studies. Hypertension 1995;26:485-90.

4. Cohn JN. Vascular wall function as a risk marker for cardiovascular disease. J Hypertens Suppl 1999;17:S41-4.

5. van Popele NM, Grobbee DE, Bots ML, Asmar R, Topouchian J, Reneman RS, et al. Association between arterial stiffness and atherosclerosis: the Rotterdam Study. Stroke 2001;32:454-60.

6. Lee $\mathrm{CJ}$, Wang JH, Chen ML, Yang CF, Chen YC, Hsu BG. Serum osteoprotegerin is associated with arterial stiffness assessed according to the cardio-ankle vascular index in hypertensive patients. J Atheroscler Thromb 2015;22:304-12

7. Shin J, Park JB, Kim Kl, Kim JH, Yang DH, Pyun WB, et al. 2013 Korean Society of Hypertension guidelines for the management of hypertension: part III-hypertension in special situations. Clin Hypertens 2015;21:3

8. Lacolley $\mathrm{P}$, Challande $\mathrm{P}$, Osborne-Pellegrin $\mathrm{M}$, Regnault V. Genetics and pathophysiology of arterial stiffness. Cardiovasc Res 2009;81:637-48.

9. Yamashina A, Tomiyama $H_{1}$ Takeda $K$, Tsuda $H$, Arai T, Hirose $K_{\text {, et }}$ al. Validity, reproducibility, and clinical significance of noninvasive brachial-ankle pulse wave velocity measurement. Hypertens Res 2002;25:359-64.

10. Kawai T, Ohishi M, Takeya Y, Onishi M, Ito N, Yamamoto K, et al. Adiponectin single nucleotide polymorphism is a genetic risk factor for stroke through high pulse wave pressure: a cohort study. J Atheroscler Thromb 2013;20:152-60.

11. Kawai T, Ohishi M, Onishi M, Ito N, Takeya Y, Maekawa Y, et al. Cut-off value of brachial-ankle pulse wave velocity to predict cardiovascular disease in hypertensive patients: a cohort study. J Atheroscler Thromb 2013:20:391-400.

12. Nakamura $M$, Yamashita $T$, Yajima J, Oikawa $Y$, Sagara $K$, Koike $A$, et al. Brachial-ankle pulse wave velocity as a risk stratification index for the short-term prognosis of type 2 diabetic patients with coronary artery disease. Hypertens Res 2010;33:1018-24.

13. Xiong Z, Zhu C, Zheng Z, Wang M, Wu Z, Chen L, et al. Relationship between arterial stiffness assessed by brachial-ankle pulse wave velocity and coronary artery disease severity assessed by the SYNTAX score. J Atheroscler Thromb 2012;19:970-6.

14. Yamashina A, Tomiyama $H_{1}$ Arai $T$, Hirose $K$, Koji $Y$, Hirayama $Y$, et al. Brachial-ankle pulse wave velocity as a marker of atherosclerotic vascular damage and cardiovascular risk. Hypertens Res 2003;26:61522.

15. Youn JC, Kim JY, Park S, Kwon J, Lee HS, Shin DH, et al. Comparison of arterial stiffness indices measured by the Colins and SphygmoCor systems. Hypertens Res 2012;35:1180-4.

16. Igarashi $Y$, Chikamori T, Tomiyama H, Usui Y, Hida S, Tanaka $H_{1}$ et al. Diagnostic value of simultaneous brachial and ankle blood pressure measurements for the extent and severity of coronary artery disease as assessed by myocardial perfusion imaging. Circ J 2005;69:237-42.

17. Tomiyama H, Koji $Y$, Yambe M, Shiina $K$, Motobe $K$, Yamada J, et al. Brachial-ankle pulse wave velocity is a simple and independent predictor of prognosis in patients with acute coronary syndrome. Circ $J$ 2005;69:815-22.

18. Vlachopoulos C, Aznaouridis K, Stefanadis C. Prediction of cardiovascular events and all-cause mortality with arterial stiffness: a systematic review and meta-analysis. J Am Coll Cardiol 2010;55:1318-27.

19. Vlachopoulos C, Aznaouridis K, Terentes-Printzios D, loakeimidis N, Stefanadis C. Prediction of cardiovascular events and all-cause mortality with brachial-ankle elasticity index: a systematic review and meta-analysis. Hypertension 2012;60:556-62.

20. Cortez-Cooper MY, Supak JA, Tanaka H. A new device for automatic measurements of arterial stiffness and ankle-brachial index. Am J Cardiol 2003;91:1519-22, A9.

21. Smith MW, Clark SP, Hutchinson JS, Wei YH, Churukian AC, Daniels $L B$, et al. A sequence-tagged site map of human chromosome 11 . Genomics 1993;17:699-725.

22. Entrez Gene: OPCML opioid binding protein/cell adhesion moleculelike [Internet]. [cited 2017 Apr 22]. [http://www.genenames.org/cgibin/gene_symbol_report?hgnc_id=HGNC:8143]

23. Entrez Gene: Proline rich 35 [Internet]. [cited 2017 Apr 22]. [https:// www.ncbi.nlm.nih.gov/gene/146325]

24. Atwood LD, Samollow PB, Hixson JE, Stern MP, MacCluer JW. Genome-wide linkage analysis of pulse pressure in Mexican Americans. Hypertension 2001;37:425-8.

25. Camp NJ, Hopkins PN, Hasstedt SJ, Coon H, Malhotra A, Cawthon $\mathrm{RM}$, et al. Genome-wide multipoint parametric linkage analysis of pulse pressure in large, extended utah pedigrees. Hypertension 2003;42:322-8. 
26. DeStefano AL, Larson MG, Mitchell GF, Benjamin EJ, Vasan RS, Li J, et al. Genome-wide scan for pulse pressure in the National Heart, Lung and Blood Institute's Framingham Heart Study. Hypertension 2004:44:152-5.

27. Sugawara J, Hayashi K, Yokoi T, Cortez-Cooper MY, DeVan AE, Anton $M A$, et al. Brachial-ankle pulse wave velocity: an index of central arterial stiffness? J Hum Hypertens 2005;19:401-6.

28. Oliver JJ, Webb DJ. Noninvasive assessment of arterial stiffness and risk of atherosclerotic events. Arterioscler Thromb Vasc Biol 2003;23:554-66.

29. Levy D, Larson MG, Benjamin EJ, Newton-Cheh C, Wang TJ, Hwang SJ, et al. Framingham Heart Study 100K Project: genome-wide associations for blood pressure and arterial stiffness. BMC Med Genet 2007;8 Suppl 1:S3.

30. Park S, Lee JY, Kim BK, Lee SH, Chang HJ, Choi D, et al. Lack of association between arterial stiffness and genetic variants by genomewide association scan. Blood Press 2015;24:258-61.

31. McEniery CM, Yasmin, Hall IR, Qasem A, Wilkinson IB, Cockcroft JR; ACCT Investigators. Normal vascular aging: differential effects on wave reflection and aortic pulse wave velocity: the Anglo-Cardiff Collaborative Trial (ACCT). J Am Coll Cardiol 2005;46:1753-60.

32. Giannattasio $C$, Failla $M$, Capra $A$, Scanziani $E_{1}$ Amigoni $M$, Boffi $L$, et al. Increased arterial stiffness in normoglycemic normotensive offspring of type 2 diabetic parents. Hypertension 2008;51:182-7.

33. Tarasov KV, Sanna S, Scuteri A, Strait JB, Orrù M, Parsa A, et al. CO$L 4 A 1$ is associated with arterial stiffness by genome-wide association scan. Circ Cardiovasc Genet 2009;2:151-8.
34. Björck HM, Länne $T$, Alehagen $U$, Persson $K$, Rundkvist L, Hamsten A et al. Association of genetic variation on chromosome 9p21.3 and arterial stiffness. J Intern Med 2009;265:373-81.

35. Sie MP, Yazdanpanah M, Mattace-Raso FU, Uitterlinden AG, Hofman $A$, Hoeks AP, et al. Genetic variation in the renin-angiotensin system and arterial stiffness. The Rotterdam Study. Clin Exp Hypertens 2009;31:389-99.

36. Mayer $0 \mathrm{Jr}$, Filipovský J, Pesta $M$, Cífková R, Dolejsová M, Simon J. The interaction of endothelial nitric oxide synthase polymorphism and current smoking in terms of increased arterial stiffness. Physiol Res 2010;59:529-36

37. Yasmin, McEniery CM, O'Shaughnessy KM, Harnett P, Arshad A, Wallace $S$, et al. Variation in the human matrix metalloproteinase- 9 gene is associated with arterial stiffness in healthy individuals. Arterioscler Thromb Vasc Biol 2006;26:1799-805.

38. Schnabel R, Larson MG, Dupuis J, Lunetta KL, Lipinska I, Meigs JB, et al. Relations of inflammatory biomarkers and common genetic variants with arterial stiffness and wave reflection. Hypertension 2008:51:1651-7.

39. Chen W, Srinivasan SR, Boerwinkle E, Berenson GS. Beta-adrenergic receptor genes are associated with arterial stiffness in black and white adults: the Bogalusa Heart Study. Am J Hypertens 2007;20:1251-7.

40. Wang K, Dickson SP, Stolle CA, Krantz ID, Goldstein DB, Hakonarson $\mathrm{H}$. Interpretation of association signals and identification of causal variants from genome-wide association studies. Am J Hum Genet 2010;86:730-42. 